\title{
ApoG2 inhibits the antiapoptotic protein, Mcl-1, and induces mitochondria-dependent apoptosis in human colorectal cancer cells
}

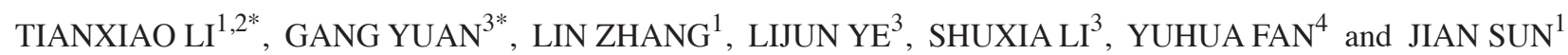 \\ ${ }^{1}$ Department of Clinical Research, Sun Yat-sen University Cancer Center, State Key Laboratory of Oncology in South China, \\ Collaborative Innovation Center for Cancer Medicine, Guangzhou, Guangdong 510060; ${ }^{2}$ Department of Pharmacy, \\ Guanghua School of Stomatology, Sun Yat-sen University, Guangzhou, Guangdong 510055; \\ Departments of ${ }^{3}$ Geriatrics and ${ }^{4}$ Neurology, The First Affiliated Hospital, \\ Sun Yat-sen University, Guangzhou, Guangdong 510080, P.R. China
}

Received November 26, 2014; Accepted August 25, 2015

DOI: $10.3892 / \mathrm{mmr} .2015 .4299$

\begin{abstract}
Colorectal cancer (CRC) is a worldwide malignancy of high incidence and mortality. At present, there is a lack of effective drugs against CRC. The B-cell leukemia/lymphoma 2 (Bcl-2) protein family members are considered to be closely associated with tumorigenesis and the chemoresistance of CRC. As a novel gossypol derivative targeting antiapoptotic proteins of the Bcl-2 family, apogossypolone (ApoG2) exhibits antitumor properties in various cancer types, although its effects against $\mathrm{CRC}$ remain to be fully elucidated. In the present study, the cytotoxicity of ApoG2 in vitro on CRC cells was investigated, with the aim of elucidating the underlying mechanism. Using an MTT assay, ApoG2 was revealed to inhibit the growth of the HT29, SW480 and HCT116 CRC cell lines in a dose- and a time-dependent manner. Hoechst staining revealed that ApoG2 induced CRC cell apoptosis, marked by morphological changes, including cell shrinkage and nuclear fragmentation. Flow cytometric analysis also detected a higher apoptotic ratio following treatment with ApoG2. The ratio was dependent upon the concentration of ApoG2, which the cells were
\end{abstract}

Correspondence to: Professor Yuhua Fan, Department of Neurology, The First Affiliated Hospital, Sun Yat-sen University, 58 Zhongshan Er Road, Guangzhou, Guangdong 510080, P.R. China

E-mail: fanyuhua@mail.sysu.edu.cn

Dr Jian Sun, Department of Clinical Research, Sun Yat-sen University Cancer Center, State Key Laboratory of Oncology in South China, Collaborative Innovation Center for Cancer Medicine, 651 Dongfeng Road East, Guangzhou, Guangdong 510060, P.R. China

E-mail: sunjian@sysucc.org.cn

${ }^{*}$ Contributed equally

Key words: apogossypolone, B-cell leukemia/lymphoma 2 family, colorectal cancer exposed to, and the duration of the exposure. Western blot analysis and immunoprecipitation experiments revealed that ApoG2 treatment led to the downregulation of the protein expression of Mcl-1, and the interruption of the binding of Mcl-1 to the protein Bax. Furthermore, treatment with ApoG2 led to the release of cytochrome $c$ into the cytoplasm and the activation of caspases 3 and 7. The present study revealed that ApoG2 inhibited the proliferation of the CRC cell lines through mitochondrial signaling pathway-dependent apoptosis, which may be associated with the disruption of the function of the Mcl-1 protein by ApoG2.

\section{Introduction}

Colorectal cancer (CRC) is a common malignancy worldwide, associated with a high incidence and a high rate of mortality (1). In particular, the number of patients diagnosed with the disease continues to rise in urban areas of China. Surgery is the most effective method for the treatment of CRC, whereas chemotherapy is also essential for achieving an improved prognosis (2). However, only a few drugs have been identified to have an active effect on CRC. The resistance of CRC to current chemotherapeutic strategies often leads to unsatisfactory outcomes, therefore, there is an urgent requirement for the development of effective drugs against CRC.

Abnormal apoptosis is a feature of cancer cells. It provides them with a means to escape regulatory checks on cell growth, and to mediate resistance to chemotherapy (3). B-cell leukemia/lymphoma 2 (Bcl-2) family proteins exert important roles in the regulation of cell apoptosis (4). Members of the $\mathrm{Bcl}-2$ protein family are divided into antiapoptotic proteins and proapoptotic proteins. Their functions differ according to their structural features. The Bcl-2 family proteins share the same four conservative sequences, termed Bcl-2 homology domains (BH1-BH4). Usually, the antiapoptotic Bcl-2 proteins feature a hydrophobic groove formed by BH1-3. They can bind to the $\mathrm{BH} 3$ domain of a proapoptotic member, and inhibit its proapoptotic function (5). Previous studies have identified that the antiapoptotic members of the protein family $\left(\mathrm{Bcl}-2, \mathrm{Bcl}-\mathrm{X}_{\mathrm{L}}\right.$ 
and Mcl-1) are widely overexpressed in a number of cancer types (6-7). Therefore, the development of drugs which mimic $\mathrm{BH} 3$ structure and inhibit the activity of antiapoptotic proteins of the Bcl-2 family provides the basis of a feasible strategy for cancer therapy.

Gossypol is a polyphenolic compound, which occurs naturally in cotton seeds and roots. It was identified as having antiproliferative activity in vitro and in vivo against several cancer types $(8,9)$. The isolated active component of gossypol, (-)-gossypol, has been assessed in phase II human clinical trials for a variety of cancer types, including chronic lymphocytic leukemia and B-cell malignancies (10-12). Apogossypolone (ApoG2) is a novel derivative of gossypol, which is synthesized by removing two aldehyde groups to achieve superior anticancer activity with less toxicity (13). ApoG2 has been reported to greatly suppress the growth of nasopharyngeal carcinoma (14), breast cancer (15), gastric carcinoma (16), hepatocellular carcinoma cells (17), prostate cancer cells (18) and lymphoma cells (19); however, the activity of ApoG2 against CRC remains to be fully elucidated.

In the present study, the antiproliferative effects of ApoG2 in CRC cells, and the ability of the compound to induce cell apoptosis, were investigated. Furthermore, the possible mechanism by which ApoG2 may induce apoptosis in CRC cells was also investigated.

\section{Materials and methods}

Cells and reagents. The human HT29, HCT116 and SW480 CRC cell lines were donated by the State Key Laboratory of Oncology in South China (Guangzhou, China). The cells were cultured in RPMI-1640 medium (Invitrogen Life Technologies, Carlsbad, CA, USA), supplemented with $10 \%$ fetal bovine serum (Invitrogen Life Technologies) and incubated at $37^{\circ} \mathrm{C}$ with $5 \% \mathrm{CO}_{2}$. ApoG2 was synthesized by Professor Dajun Yang (Ascenta Therapeutics, Inc.,Malvern, PA, USA) and dissolved in pure dimethyl sulfoxide (DMSO; MP Biomedicals, Solon, OH, USA) with a stock concentration of $20 \mathrm{mmol} / 1$, prior to storage at $-20^{\circ} \mathrm{C}$. The 3-[4,5-dimethylthiazol-2-thiazolyl]-2,5-diphenyltetrazolium bromide (MTT) reagent, for use in subsequent assays, was purchased from MP Biomedicals.

MTT assay. The antiproliferative effects of ApoG2 on the three CRC cell lines (HT29, HCT116 and SW480) were determined using an MTT assay. A total of $10^{4}$ cells were seeded into 96-well plates and were incubated overnight. The cells were treated with ApoG2 at concentrations of $1.25,2.5$ or $5 \mu \mathrm{M}$ for durations of 24,48 or $72 \mathrm{~h}$. Subsequently, $20 \mu 15 \mathrm{mg} / \mathrm{ml}$ MTT was added to each well and incubated for a further $4 \mathrm{~h}$. The supernatant was carefully discarded and $200 \mu \mathrm{l}$ DMSO was added to dissolve the formazan sediment. The absorbance was measured using a microplate reader (V-530; JASCO International, Co., Ltd., Tokyo, Japan) at a wavelength of $490 \mathrm{~nm}$. All experiments were replicated in triplicate. Tumor cells, which were cultured in the complete medium with $0.1 \%$ DMSO, were used as a control, and wells containing only complete medium with $0.1 \%$ DMSO served as a blank control. The inhibition of cell growth was measured as the percentage of viable cells relative to the control, and calculated as: [Optical density $\left.\left.(\mathrm{OD})_{\text {treated }}-\mathrm{OD}_{\text {blank }}\right] /\left(\mathrm{OD}_{\text {control }}-\mathrm{OD}_{\text {blank }}\right)\right] \times 100$.

Western blot assay. The total cell lysates were harvested and electrophoresed in $10 \%$ or $15 \%$ sodium dodecyl sulfate (SDS)-polyacrylamide gels (Sigma-Aldrich, St. Louis, MO, USA). The proteins were transferred onto polyvinylidene difluoride (PVDF) membranes (Roche, Basel, Switzerland). The transferred PVDF membranes were subsequently blocked with $5 \%$ non-fat milk for $1 \mathrm{~h}$ at room temperature. Western blotting was performed with primary antibodies raised against Bcl-2 (cat. no. \#2870; Cell Signaling Technology, Inc., Beverly, MA, USA), Bcl-X (cat. no. \#2762; Cell Signaling Technology, Inc.), Mcl-1 (cat. no. \#5354; Cell Signaling Technology, Inc.), Bax (cat. no. sc-23959; Santa Cruz Biotechnology, Inc., Santa Cruz, CA, USA), Bak (cat. no. \#6947; Cell Signaling Technology, Inc.), cytochrome $c$ (cat. no. \#4272; Cell Signaling Technology, Inc.) and glyceraldehyde-3-phopsphate dehydrogenase (GAPDH; cat. no. \#2118; Cell Signaling Technology, Inc.), followed by incubation with secondary antibodies conjugated to horseradish peroxidase (cat. nos. \#7074 and \#7076; Cell Signaling Technology, Inc.). The proteins were detected through film development using a clarity-enhanced chemiluminescence reagent (cat. no. \#170-5056; Bio-Rad Laboratories, Hercules, CA, USA).

Immunoprecipitation analysis. Protein immunoprecipitation was performed to determine protein interactions among the Bcl-2 family members. The cell lysates were initially incubated with antibodies raised against Bax (cat. no. sc-23959; Santa Cruz Biotechnology, Inc.) for $1 \mathrm{~h}$ at $4^{\circ} \mathrm{C}$, prior to mixing with protein A/G agarose beads (sc-2001, Santa Cruz Biotechnology, Inc.) and agitating the solution overnight at $4^{\circ} \mathrm{C}$. The proteins bound to the beads were collected by centrifugation $(2,000 \mathrm{x} \mathrm{g}$, $10 \mathrm{sec}, 4^{\circ} \mathrm{C}$ ) and subsequently eluted using denaturing loading buffer (Pierce 26149, Co-Immunoprecipitation kit; Pierce Biotechnology, Rockford,IL, USA). The antiapoptotic proteins, which had interacted with Bax were identified by western blotting using antibodies against antiapoptotic proteins, according to the method described previously (14).

Detection of cells undergoing apoptosis. A total of $10^{5}$ cells were plated into six-well plates and were cultured overnight. The cells were subsequently incubated with $0.1 \%$ DMSO and $1.25,2.5$ or $5 \mu \mathrm{mol} / 1$ ApoG2 for $48 \mathrm{~h}$. Following treatment, the cells were fixed in mounting medium (P0126, Beyotime Institute of Biotechnology, Haimen, China) for $10 \mathrm{~min}$ at room temperature, rinsed twice with phosphate-buffered saline (PBS; 3 min each wash) and subsequently stained with $5 \mu \mathrm{g} / \mathrm{ml}$ Hoechst 33258 for $5 \mathrm{~min}$. The cells were rinsed twice with PBS and any nuclei changes resulting from apoptosis were observed by fluorescence microscopy (DFC480; Leica Microsystems CMS GmbH, Mannheim, Germany).

Flow cytometric analysis. Cell apoptosis was also assessed by flow cytometry (Beckman Coulter, Fullerton, CA, USA) using annexin V-fluorescein isothiocyanate (FITC) and propidium iodide stain (PI) (cat. no. 556405, Apoptosis Detection kit; BD Biosciences, Franklin Lakes, NJ, USA). ApoG2-treated cells were harvested, centrifuged at $800 \mathrm{x} g$ for $5 \mathrm{~min}$ at room temper- 
A

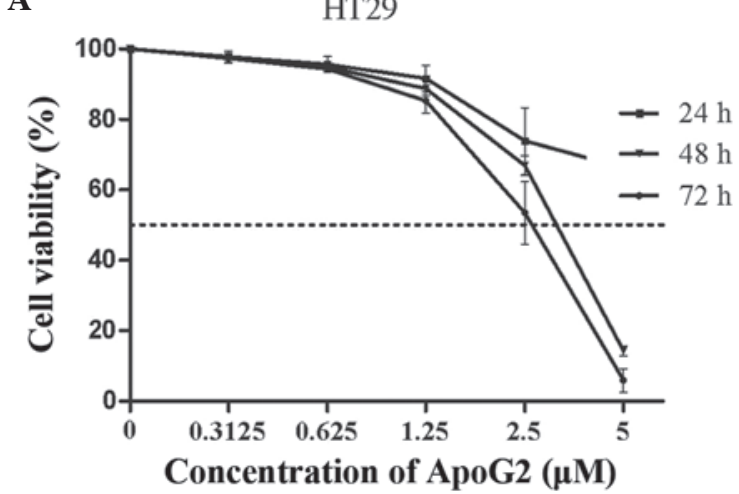

C

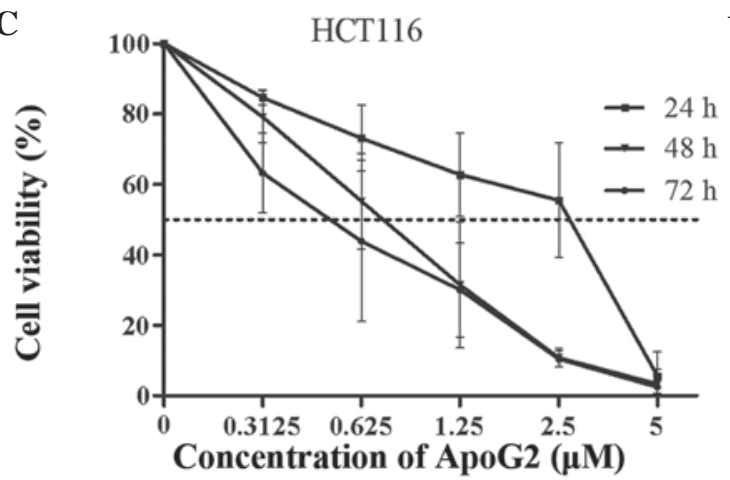

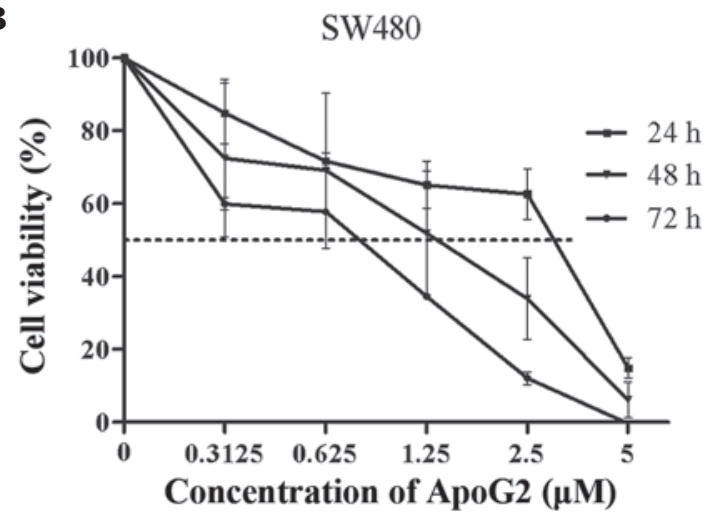

D

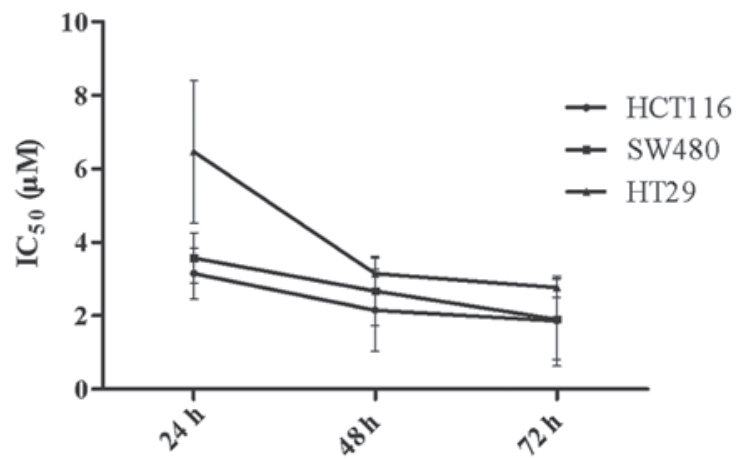

Figure 1. Growth-inhibitory effects of ApoG2 on the human (A) HT29, (B) SW480 and (C) HCT116 CRC lines. The growth-inhibitory effect of ApoG2 on the three human CRC cell lines was determined using an MTT assay. Cell viability data are expressed as the mean \pm standard error of the mean for three independent experiments. (D) The $\mathrm{IC}_{50}$ values are shown as predictive values, with a $95 \%$ confidence interval for three independent experiments. ApoG2, apogossypolone; $\mathrm{IC}_{50}$, concentration of inhibitor required to effect $50 \%$ inhibition; CRC, colorectal cancer.

ature, washed with PBS, centrifuged as before and resuspended in $500 \mu \mathrm{l}$ binding buffer, containing $5 \mu \mathrm{l}$ annexin V-FITC and $5 \mu \mathrm{l}$ PI. The cells were incubated in the dark in staining solution (cat. no. C0003, Beyotime Institute of Biotechnology) for $15 \mathrm{~min}$. The level of apoptosis of the cells was analyzed using the flow cytometric system with ModFit LT for windows Trial and Reader Version 3.2 (Verity Software House, Topsham, ME, USA).

Cytochrome c release assay. To assess the release of cytochrome $c$ from mitochondria into the cytoplasm, the cytosolic and mitochondrial fractions were isolated using a mitochondria/cytosol fractionation kit (cat. no. \#K256-25; Biovision, Tuczon, AZ, USA), according to the manufacturer's instructions. The cells were collected, washed with PBS and suspended in $1 \mathrm{ml} 5 \mathrm{X}$ dilution of cytosolic extraction solution (K256-100; BioVision Inc., Milpitas, CA, USA) on ice for $10 \mathrm{~min}$, prior to repeatedly beating the cells with a syringe to destroy the cytomembranes. The cellular and nuclear debris were removed by centrifuging the homogenates twice at $700 \mathrm{x}$ g for $10 \mathrm{~min}$ at $4^{\circ} \mathrm{C}$. The supernatants were pelleted again at $10,000 \times \mathrm{g}$ for $30 \mathrm{~min}$ at $4^{\circ} \mathrm{C}$, and the supernatants were subsequently collected as the cytosolic fraction. The quantity of cytochrome $c$ in the cytosolic fraction was determined by western blotting, as described above.

Detection of the cleavage of caspases 3 and 7. Western blotting was used to identify the cleavage of the caspase family proteins, caspase 3 and 7. The total cell proteins were extracted and separated by electrophoresis on $15 \%$ SDS-polyacrylamide gels and transferred onto PVDF membranes. Following blocking in 5\% non-fat milk, the PVDF membranes were incubated with primary antibodies raised against caspase 3 (cat. no. \#9665; Cell Signaling Technology, Inc.), caspase 7 (cat. no. \#9492; Cell Signaling Technology, Inc.) and GAPDH (cat. no. \#2118; Cell Signaling Technology, Inc.), prior to an incubation with secondary antibodies conjugated to horseradish peroxidase. The procaspase and cleaved caspase protein bands were detected using the clarity-enhanced chemiluminescence reagent (Bio-Rad Laboratories), as described previously.

Statistical analysis. All data are expressed as the mean \pm standard error of mean for three independent experiments. The mean was compared with the one-way analysis of variance using SPSS 17.0 software (SPSS, Inc., Chicago, IL, USA). $\mathrm{P}<0.05$ was considered to indicate a statistically significant difference.

\section{Results}

ApoG2 inhibits the proliferation of the different CRC cell lines. An MTT assay was performed to investigate the growth-inhibitory effects of ApoG2 on the three CRC cell lines. As shown in Fig. 1, ApoG2 inhibited the growth of the HT29, SW480 and HCT116 cells in a time- and a dose-dependent manner. ApoG2 exerted a more pronounced effect on the SW480 and HCT116 cells compared with the HT29 cells. The concentration of ApoG2 required for a half 
maximal inhibitory concentration $\left(\mathrm{IC}_{50}\right)$ of the HT29 cells at 48 and $72 \mathrm{~h}$ was 3.12 and $2.57 \mu \mathrm{mol} / 1$, respectively. The HT29 cells failed to respond to ApoG2 treatment following a period of $24 \mathrm{~h}$. By contrast, the $\mathrm{IC}_{50}$ values of ApoG2 for the SW480 cells at 24,48 and $72 \mathrm{~h}$ were $4.04,1.44$ and $0.75 \mu \mathrm{mol} / \mathrm{l}$, respectively, and those for the HCT116 cells were 3.46, 0.77 and $0.57 \mu \mathrm{mol} / 1$, respectively.

Protein expression levels for the Bcl-2 family members in the $C R C$ cells. Since the Bcl-2 family proteins are possible targets of ApoG2, the protein expression levels of the Bcl-2 family proteins (Bcl-2, Bcl-X $\mathrm{L}_{\mathrm{L}}, \mathrm{Mcl}-1$, Bax and Bak) in CRC cells were investigated. The nasopharyngeal cancer cell line, CNE-1, in which Bcl-2 family proteins are highly expressed, was used as a positive control. As shown in Fig. 2, high protein expression levels of the antiapoptotic proteins, $\mathrm{Bcl}-2$ and $\mathrm{Bcl}-\mathrm{X}_{\mathrm{L}}$, were obtained in the HT29, HCT116 and SW480 cell lines, whereas the expression of the antiapoptotic protein, Mcl-1, differed among these three CRC cell lines. The proapoptotic proteins, Bax and Bak, were only highly expressed in HT29 cells compared with CNE-1.

ApoG2 affects the protein expression levels of the Bcl-2 family. Western blot analysis was performed to observe the changes in the protein expression levels of Bcl-2 family members following treatment with serial concentrations of ApoG2 for $48 \mathrm{~h}$. As shown in Fig. 3A, the protein expression levels of the antiapoptotic protein, Mcl-1, were markedly decreased upon treatment with ApoG2, and the effect observed was more pronounced in the SW480 and HCT116 cells compared with the HT29 cells. The relative protein expression levels compared with the control group following quantification are shown in Fig. 3B.

ApoG2 disrupts the interactions between the Bcl-2 family proteins. To further assess whether the binding of the Mcl-1 protein to Bax was altered by treatment with ApoG, immunoprecipitation assays were performed. As shown in Fig. 3C, in the untreated CRC cells, Mcl-1 and Bax, were bound to each other (lane 1); however, when the cells were treated with $2.5 \mu \mathrm{mol} / 1$ ApoG2 for $48 \mathrm{~h}$, the Mcl-1/Bax binding in all three CRC cells was completely disrupted (lane 2). Lane 3 was loaded with homologous immunoglobulin $\mathrm{G}$ and the anti-Bax antibody as a negative control.

ApoG2 induces apoptosis in the CRC cells. Hoechst 33258 staining and flow cytometry were used to assess ApoG2-induced apoptosis in the CRC cells. The nuclear morphological changes of the cells exposed to ApoG2, as revealed by Hoechst 33258 staining and observed by fluorescence microscopy, is shown in Fig. 4A. ApoG2-treated cells exhibited clear apoptotic characteristics, including cell shrinkage and nuclear fragmentation. The results of the flow cytometric analysis shown in Fig. 4B also indicated that ApoG2 promoted cell apoptosis at $48 \mathrm{~h}$. The percentages of apoptotic cells increased with the concentration of ApoG2. The level of apoptosis induced by ApoG2 was also assessed over time. As shown in Fig. 4C, apoptosis occurred as early as $24 \mathrm{~h}$ following the treatment with ApoG2, and the apoptotic rate increased concomitantly with the duration of the treatment. The percentages of the cells undergoing apoptosis

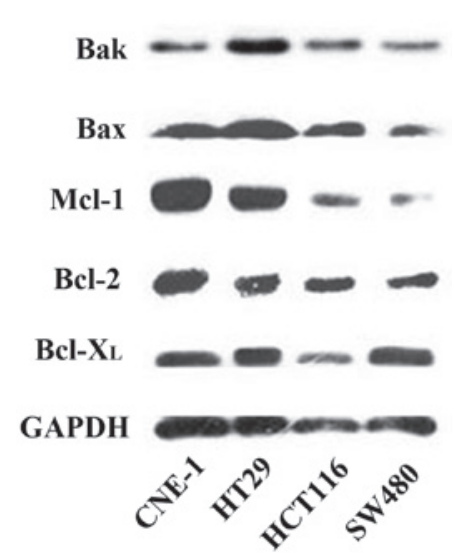

Figure 2. Protein expression levels of the Bcl-2 protein family members in human CRC cells. The total cell protein was extracted from the human HT29, HCT116 and SW480 CRC cell lines, and from CNE-1 cells, as a positive control. Western blotting revealed the protein expression levels of Bcl-2, Bcl-X $\mathrm{L}$, Mcl-1, Bax and Bak. GAPDH was used as a loading control. GAPDH, glyceraldehyde-3-phosphate dehydrogenase; CRC, colorectal cancer.

for the three cell lines, and at the different time points following treatment with ApoG2, are shown in Table I.

ApoG2 promotes cytochrome c release. Since the release of cytochrome $c$ from the mitochondria into the cytosol is usually indicative of apoptosis, whether ApoG2-induced apoptosis of the CRC cells involved the release of cytochrome $c$ was investigated. As shown in Fig. 5A, an investigation of the levels of cytochrome $c$ in the cytosol revealed that cytochrome $c$ was almost undetectable in the cytosol of untreated CRC cells (leftmost lane in each gel). Following treatment of the cells with ApoG2 at the indicated concentrations for $48 \mathrm{~h}$, cytochrome $c$ was translocated into the cytosol in a concentration-dependent manner (lanes 2-4 of each gel).

ApoG2 induces the activation of caspase 3 and 7. Caspase 3 is one of the executioners of apoptosis, and it is the first caspase to be activated by initiator cleavage. The activation of caspase 3 provides a marker of cell apoptosis. To confirm that ApoG2-promoted apoptosis signaling was occurring in CRC cells, the activation of caspase 3 and caspase 7 was investigated. Caspase 7 is normally activated subsequently to caspase 3. As shown in Fig. 5B, the levels of the cleaved caspases 3 and 7 increased significantly following treatment of ApoG2 at the indicated concentrations at $48 \mathrm{~h}$.

\section{Discussion}

The absence of effective chemotherapeutic drugs often leads to a poor prognosis of CRC. It is a major concern that CRC is becoming resistant to treatment with the drugs currently used, including 5-fluorouracil and oxaliplatin. Members of the Bcl-2 protein family are considered to be associated with tumorigenesis and the resistance of CRC to chemotherapy. Prabhudesai et al (20) revealed that increased expression levels of the antiapoptotic proteins, and reduced expression levels of Bax, are associated with poor responses to chemotherapy and shorter overall survival rates in $\mathrm{CRC}$, indicating that the 


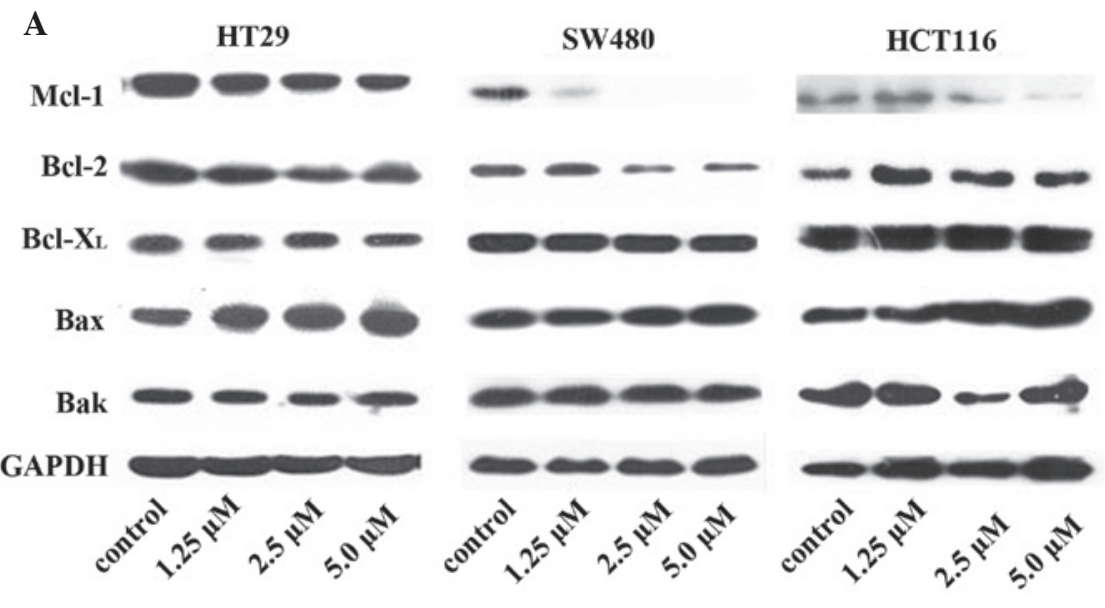

B

HT29

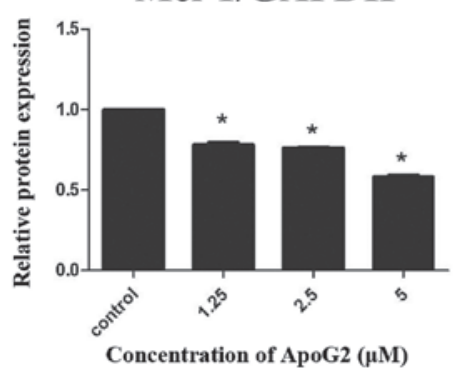

SW480

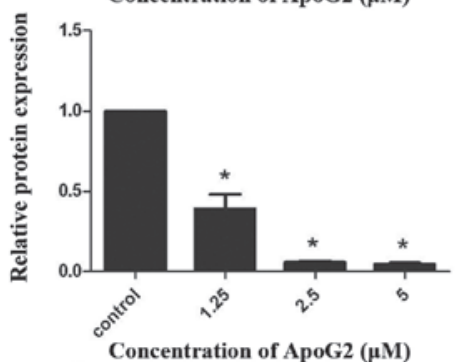

HCT116

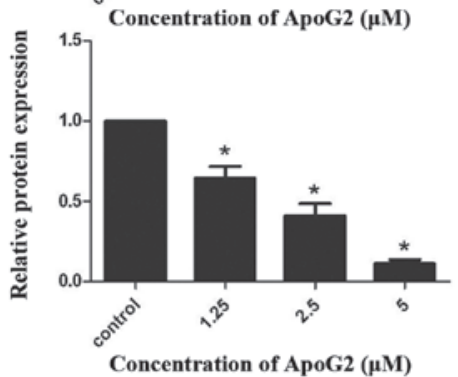

C

HT29

\section{IP:Bax}
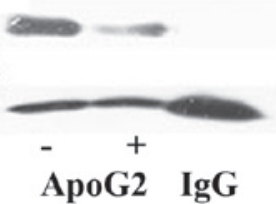

SW480

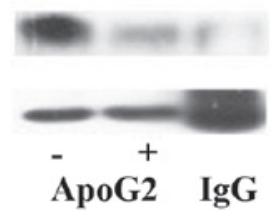

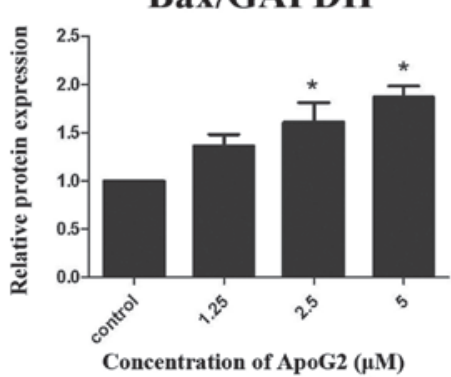
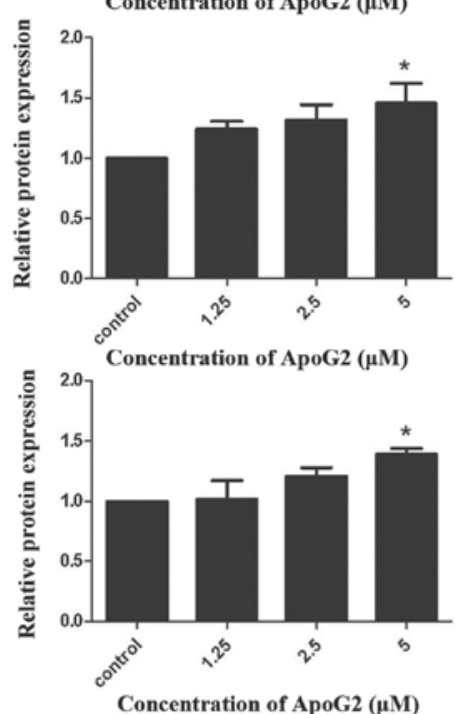

HCT116

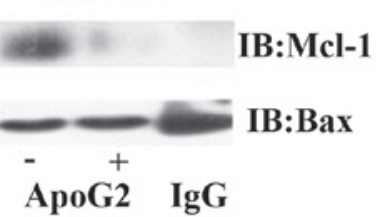

Figure 3. Effects of ApoG2 on the expression levels and function of the Bcl-2 family proteins in human CRC cell lines. (A) Western blot analysis was performed using specific antibodies against Bcl-2, Bcl- $\mathrm{X}_{\mathrm{L}}, \mathrm{Mcl}-1$, Bax and Bak. GAPDH was used as a control to normalize each lane as the loading control. (B) Densitometric quantitative results of the protein expression levels of the target proteins normalized against GAPDH. The effect of ApoG2 on target protein levels is presented as the fold of protein expression compared with the control vehicle-treated cells, which are expressed as 1-fold. The height of the bars indicate the mean of three independent experiments, while the error bars indicate the standard error of mean $\left({ }^{*} \mathrm{P}<0.05\right.$, compared with the control). $(\mathrm{C}) \mathrm{Cell}$ lysates were immunoprecipitated with primary anti-Bax antibodies. Western blots were performed to detect the quantity of Mcl-1 protein binding to Bax. GAPDH, glyceraldehyde-3-phosphate dehydrogenase; ApoG2, apogossypolone; IP, immunoprecipitate; IB, immunoblot; CRC, colorectal cancer.

$\mathrm{Bcl}-2$ protein family is involved in tumorigenesis and the resistance of CRC to chemotherapies.
ApoG2 is a non-peptide molecular inhibitor of the antiapoptotic Bcl-2 family of proteins, derived from gossypol. Compared 
A
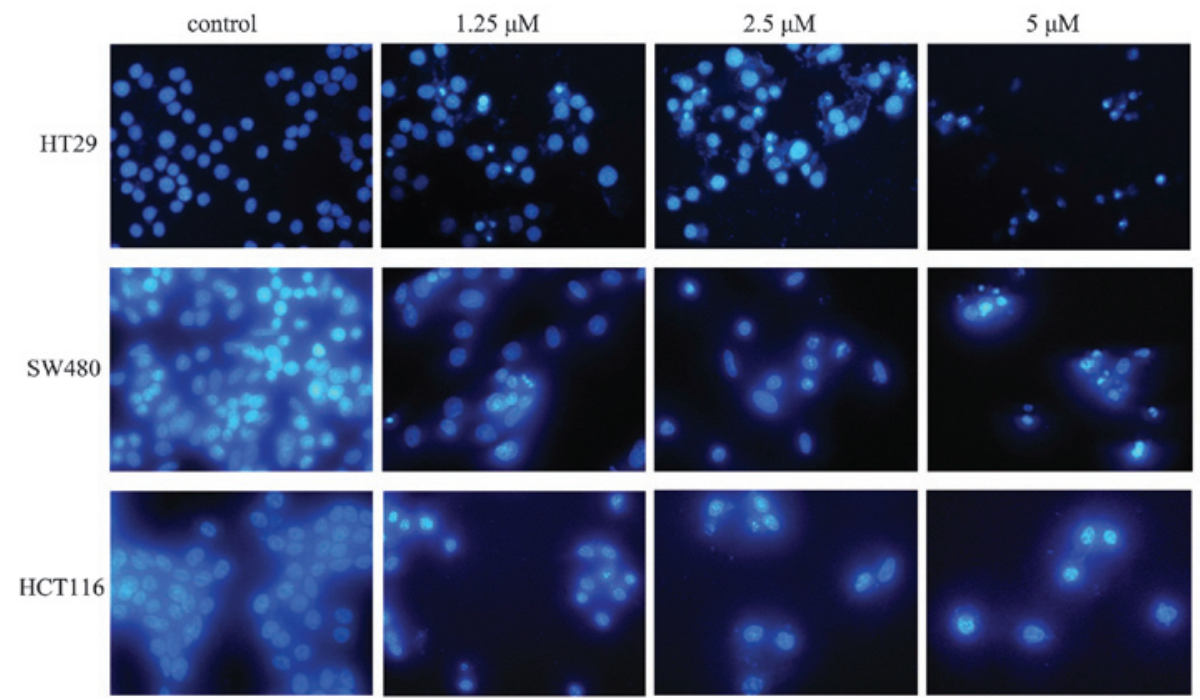

B
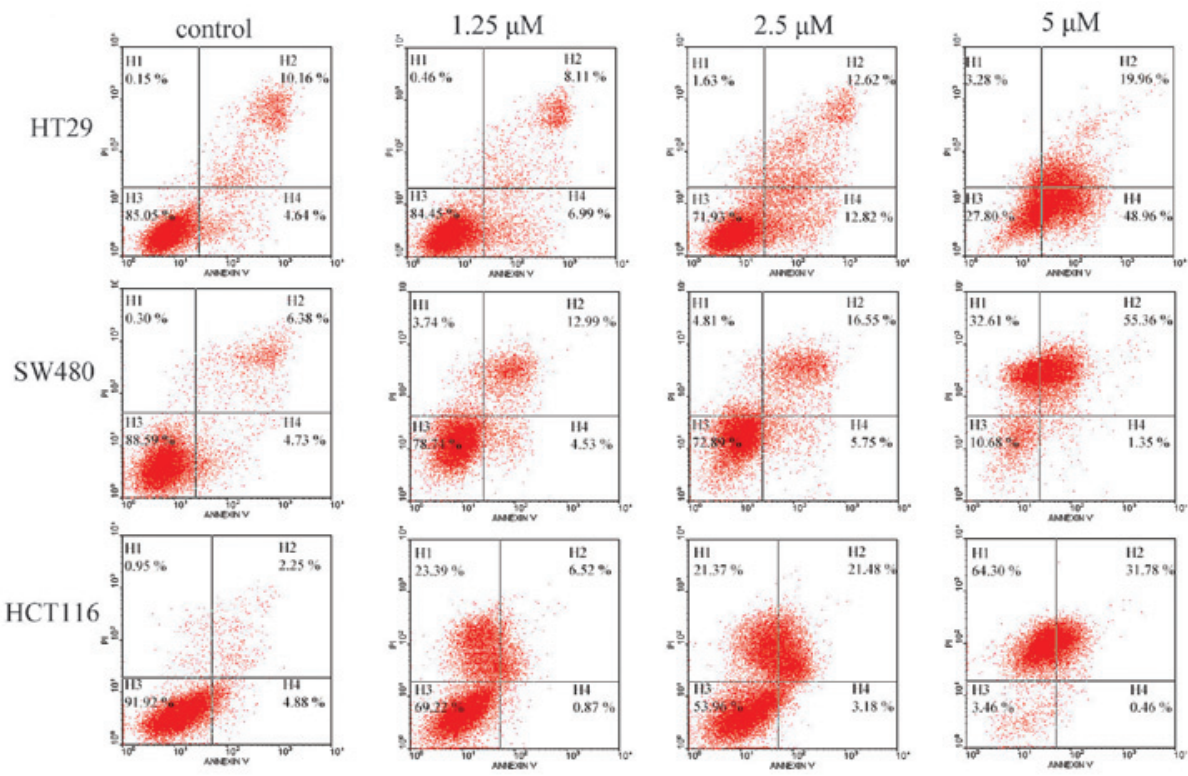

C
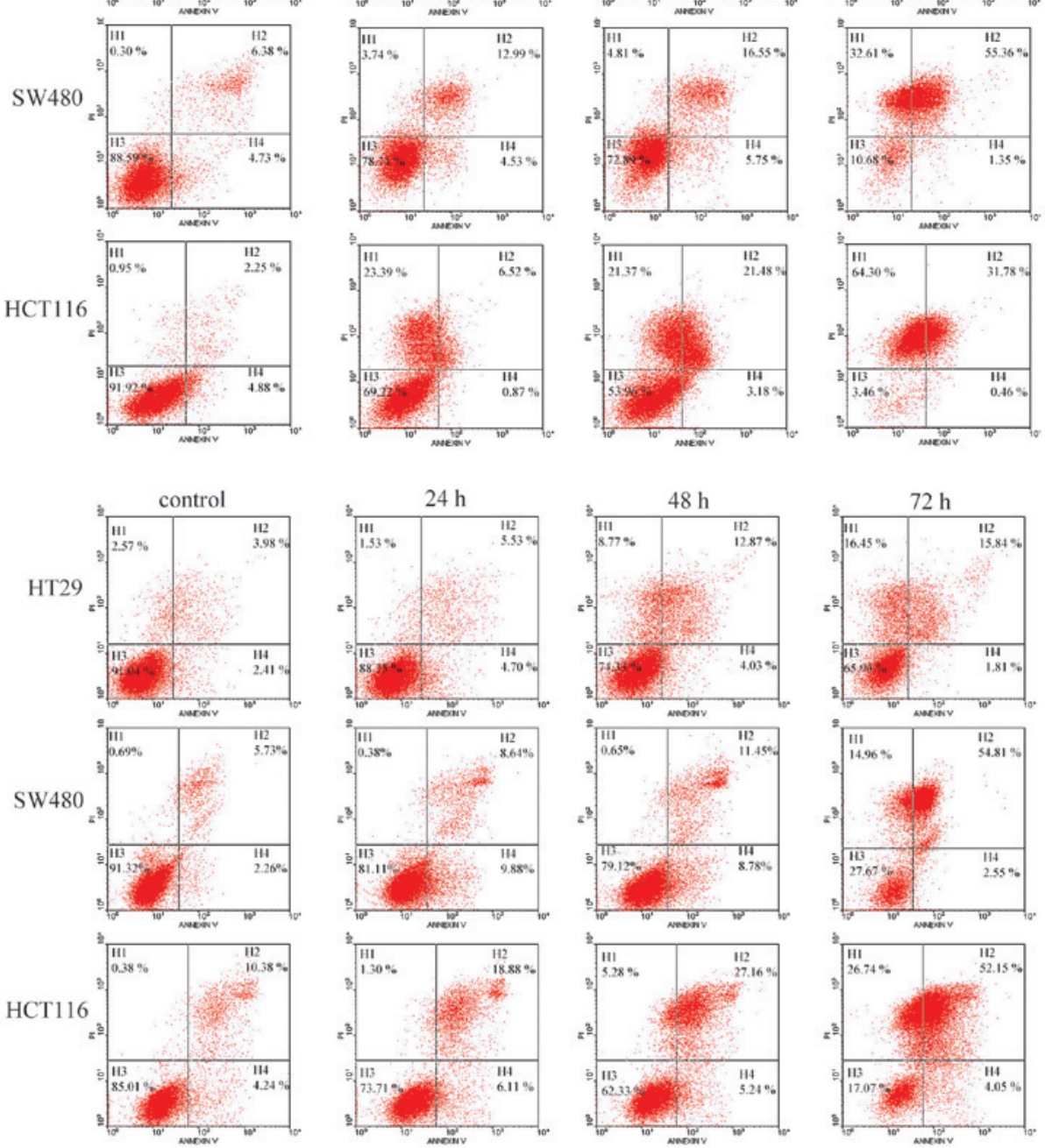

Figure 4. Cell apoptosis of the human CRC cell lines induced by ApoG2. (A and B) The cells were treated with the indicated concentrations of ApoG2 for $48 \mathrm{~h}$, (A) The fluorescence microscopic observation of the Hoechst 33258-stained cells. (B) The apoptotic levels of the cells was analyzed by flow cytometry following labeling of the cells with annexin V/FITC and PI as markers for apoptosis. (C) The cells were stained with annexin V/FITC and PI, and were analyzed by flow cytometry following treatment with $2.5 \mu \mathrm{mol} / 1 \mathrm{ApoG} 2$ for 24,48 and $72 \mathrm{~h}$. ApoG2, apogossypolone; FITC, fluorescein isothiocyanate; PI, propidium iodide; CRC, colorectal cancer. 
Table I. Percentage of apoptotic cells induced by treatment with ApoG2.

A, Cells treated with ApoG2 at different concentrations

\begin{tabular}{lrrrr} 
& & \multicolumn{3}{c}{ Concentration of ApoG2 $(\mu \mathrm{M})$} \\
\cline { 3 - 5 } Cell line & \multicolumn{1}{c}{ Control } & \multicolumn{1}{c}{1.25} & 2.5 & 5 \\
\hline HT29 & $14.80 \pm 1.33$ & $15.27 \pm 0.50$ & $25.06 \pm 1.16^{\mathrm{a}}$ & $67.97 \pm 4.54^{\mathrm{b}}$ \\
SW480 & $11.31 \pm 0.52$ & $17.41 \pm 0.80$ & $22.65 \pm 1.34^{\mathrm{a}}$ & $55.88 \pm 3.01^{\mathrm{b}}$ \\
HCT116 & $7.13 \pm 0.93$ & $7.46 \pm 0.56$ & $24.65 \pm 2.27^{\mathrm{b}}$ & $32.14 \pm 0.93^{\mathrm{b}}$ \\
\hline
\end{tabular}

B, Cells treated with ApoG2 for different durations

\begin{tabular}{lcccc}
\hline & & \multicolumn{3}{c}{ Duration of treatment (h) } \\
\cline { 3 - 5 } Cell line & \multicolumn{1}{c}{ Control } & 24 & 48 & 72 \\
\hline HT29 & $6.40 \pm 1.13$ & $10.67 \pm 0.92$ & $16.83 \pm 1.11^{\mathrm{a}}$ & $18.27 \pm 1.36^{\mathrm{b}}$ \\
SW480 & $8.26 \pm 0.92$ & $18.58 \pm 0.99$ & $20.63 \pm 1.34^{\mathrm{a}}$ & $56.38 \pm 2.93^{\mathrm{b}}$ \\
HCT116 & $13.72 \pm 1.94$ & $21.95 \pm 5.58$ & $31.05 \pm 2.31^{\mathrm{a}}$ & $57.11 \pm 2.18^{\mathrm{b}}$ \\
\hline
\end{tabular}

Quantification of the apoptotic cells, as analyzed by flow cytometric analyses. The proportions of apoptotic cells undergoing apoptosis were measured as the accumulations of cells distributed in the lower right and upper right quadrants, representing early apoptotic and necrotic/late apoptotic cells, respectively. The data are expressed as the mean \pm standard error of the mean of three independent experiments. ${ }^{\mathrm{a}}<0.05$ and ${ }^{\mathrm{b}} \mathrm{P}<0.01$, compared with the control group. ApoG2, apogossypolone.

A

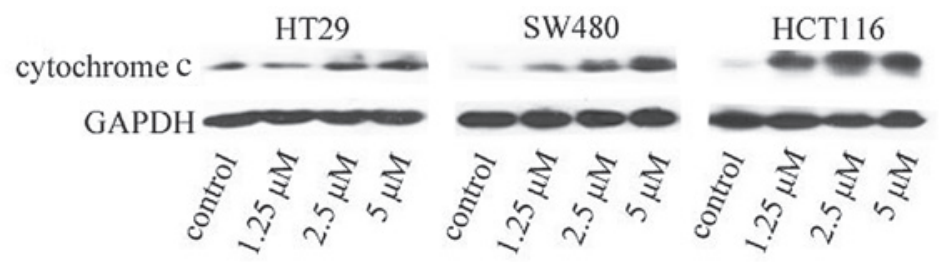

B

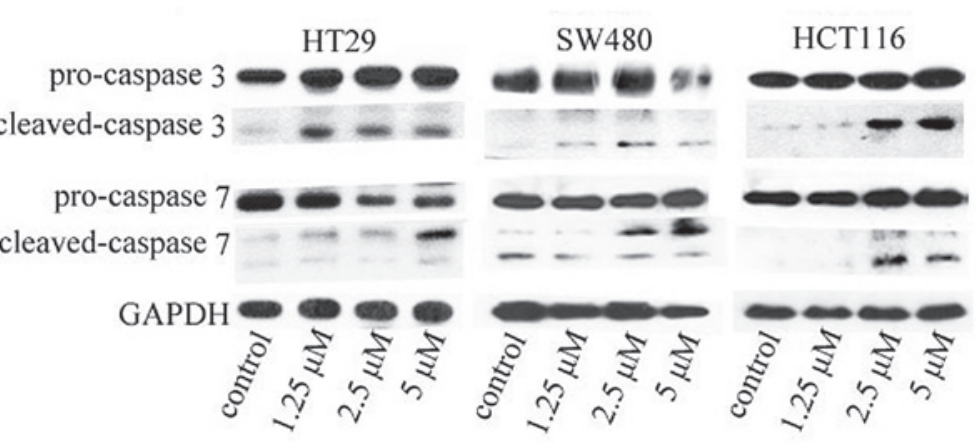

Figure 5. Activation of apoptosis mediated by the mitochondrial signaling pathway by ApoG2 in human colorectal cancer cell lines. The cells were treated with control vehicle $0.1 \%$ dimethyl sulfoxide) and the indicated concentrations of ApoG 2 for $48 \mathrm{~h}$. (A) The proteins obtained from the cytosolic fractions were separated by $15 \%$ SDS-PAGE and detected using an anti-cytochrome $c$ antibody. (B) The cell lysates were subjected to $15 \%$ SDS-PAGE gel electrophoresis and detected using caspase-3 and caspase-7 antibodies. GADPH, glyceraldehyde-3-phosphate dehydrogenase.

with gossypol, ApoG2 retains the identical antitumor effects, while having less toxicity. As previously reported, ApoG2 was well tolerated by healthy mice, and caused no toxic side-effects when administered to normal tissues, to gastric xenografts or to hepatocellular carcinoma xenografts at antitumor doses $(16,21)$. ApoG2 exhibits a similar action to cis-platinum in cancer cells in nasopharyngeal carcinoma (14), or to CHOP chemotherapy (cyclophosphamide, hydoxydaunorubicin, Oncovin ${ }^{\circledR}$ and prednisone in combination) in large-cell lymphoma and prednisolone in combination] (22). Numerous previous studies have confirmed the antitumor effects of ApoG2 and its safe administration in a variety of cancer models (14-19).

It was reported that gossypol exerts a cytotoxic effect on CRC cells (23). Furthermore, CRC cells were more sensitive to gossypol compared with other human cancer cell lines, including erythroleukemia and mammary adenocarcinoma (24). 
Consequently, it may be surmised that anti-Bcl-2 agents may provide an alternative to well-established CRC therapies. As an inhibitor of the Bcl-2 family proteins, ApoG2 is therefore a promising candidate.

Three CRC cell lines, which exhibit different degrees of differentiation (HT29, HCT116 and SW480), were selected as models to investigate CRC in vitro. The results of the MTT assay revealed that the poorly differentiated cell lines, HCT116 and SW480, were markedly more sensitive to ApoG2 compared with the highly differentiated cell line, HT29, suggesting that ApoG2 may be more effective as a therapy for poorly differentiated cancers. As observed in the western blot analysis, the HT29 cells expressed higher levels of the antiapoptotic proteins, Bcl-2, Bcl- $\mathrm{X}_{\mathrm{L}}$ and Mcl-1, compared with the HCT116 and SW480 cell lines, consistent with a previous study, which reported the association of the protein expression of $\mathrm{Bcl}-2$ with the degree of tumor differentiation (25).

ApoG2 is reported to be a pan-inhibitor of the Bcl-2 family of antiapoptotic proteins, including $\mathrm{Bcl}-2, \mathrm{Bcl}_{\mathrm{L}}$ and $\mathrm{Mcl}-1$ (26). ApoG2 binds to recombinant $\mathrm{Bcl}-2, \mathrm{Bcl}-\mathrm{X}_{\mathrm{L}}$ and $\mathrm{Mcl}-1$ proteins with $K_{\mathrm{i}}$ values of 35,660 and $25 \mathrm{nmol} / 1$, respectively, suggesting that ApoG2 has the highest affinity for Mcl-1 among the antiapoptotic Bcl-2 family proteins (22). Antiapoptotic proteins of the Bcl-2 family are therefore potential targets of ApoG2, as demonstrated in other cancer models. In the present study, the effects of ApoG2 on the expression and interaction of $\mathrm{Bcl}-2$ protein family members in CRC cells was investigated. ApoG2 disrupted the binding of Mcl-1 to Bax in all three CRC cell lines investigated. The sensitivity of the CRC cell lines to ApoG2 correlated with the extent to which Mcl-1 was inhibited, indicating that the anti-CRC effect of ApoG2 may be associated with its antagonism to Mcl-1. ApoG2 treatment also led to a reduction in the protein expression level of Mcl-1, concomitantly with an increased protein expression of Bax, suggesting that the antagonistic effects of ApoG2 on Mcl-1 released Bax protein from the suppressive action of Mcl-1. Differently from the effects of ApoG2 reported previously in other types of cancer cells, Mcl-1 protein, and not $\mathrm{Bcl}-2$ or $\mathrm{Bcl}-\mathrm{X}_{\mathrm{L}}$, appears to be the predominant target of ApoG2 in CRC cells.

The apoptosis-inducing effects of ApoG2 in CRC cells were investigated in the present study. As expected, CRC cells treated with ApoG2 exhibited clear apoptotic characteristics, including cell shrinkage and nuclear fragmentation. The flow cytometric analysis also revealed higher percentages of cells undergoing apoptosis upon treatment with ApoG2. In addition, the ApoG2-inducing apoptosis effects were investigated over time. As determined from the viability of the cells using an MTT assay, the number of apoptotic cells increased upon treatment with higher concentrations of ApoG2 or for a prolonged time period, suggesting that ApoG2 inhibited the proliferation of CRC cells by inducing cell apoptosis.

In the present study, ApoG2 inhibited Mcl-1/Bax binding and led to an increase in the protein expression of Bax. Cell apoptosis was also observed following ApoG2 treatment. It is well established that the Bcl-2 protein family regulates cell apoptosis predominantly through the mitochondrial pathway. The antagonistic action of antiapoptotic proteins of the Bcl-2 family facilitates the penetration of proapoptotic proteins into the mitochondrial membrane, and the subsequent release of cytochrome $c$ into the cytoplasm, in order to acti- vate caspase family proteins and to initiate cell apoptosis (5). Since ApoG2 disrupted Mcl-1/Bax binding in the CRC cells, it was surmised that ApoG2 may stimulate the release of cytochrome $c$ to initiate cell apoptosis. As expected, higher levels of cytochrome $c$ in the cytoplasm were observed following ApoG2 treatment. The release of cytochrome $c$ into the cytoplasm is a common feature of mitochondrial-dependent apoptosis. The results in the present study indicated that ApoG2 may induced apoptosis in CRC cells through the mitochondrial signaling pathway. Subsequently, the activation of the caspase proteins was assessed. Caspase 3 is one of the key executioners of apoptosis activated by upstream apoptotic signaling molecules, including cytochrome $c$. Caspase 7 is another executioner of apoptosis, activated after caspase 3. The activation of caspases 3 and 7 by ApoG2 in confirmed that cell apoptosis was induced by ApoG2. In addition to the disruption of the activity of $\mathrm{Mcl}-1$, the protein expression levels of Mcl-1 were decreased upon ApoG2 treatment in CRC cells. A previous study reported that the Mcl-1 protein was downregulated by caspase 3 in response to tumor necrosis factor-related apoptosis-inducing ligand-induced apoptosis in leukemic T cells (27). Since the present study revealed that ApoG2 activated caspase 3 in CRC cells, this may offer an explanation for the decreased expression of Mcl-1 following ApoG2 treatment. An accumulating body of evidence supports that Mcl-1 is associated with colorectal carcinogenesis (28-29), whereas the role of $\mathrm{Bcl}-2$ in colorectal carcinoma remains controversial, and remains to be fully elucidated (30). Since ApoG2 inhibited the protein expression and the function of Mcl-1, it may potentially be of use in colorectal cancer therapy.

In conclusion, the present study identified that ApoG2 exerts marked antiproliferative effects on human colorectal cancer cells, which is at least partly dependent on preventing the Bcl-2 family protein member, Mcl-1, from binding to the proapoptotic protein, Bax, resulting in the induction of mitochondrial signaling pathway-dependent apoptosis.

\section{References}

1. Jemal A, Bray F, Center MM, Ferlay J, Ward E and Forman D: Global cancer statistics. CA Cancer J Clin 61: 69-90, 2011.

2. André T, Boni C, Navarro M, Tabernero J, Hickish T, Topham C, Bonetti A, Clingan P, Bridgewater J, Rivera F and de Gramont A: Improved overall survival with oxaliplatin, fluorouracil and leucovorin as adjuvant treatment in stage II or III colon cancer in the MOSAIC trial. J Clin Oncol 27: 3109-3116, 2009.

3. Reed JC: Dysregulation of apoptosis in cancer. Cancer J Sci Am 4 (Suppl 1): S8-S14, 1998.

4. Adams JM and Cory S: The Bcl-2 protein family: Arbiters of cell survival. Science 281: 1322-1326, 1998.

5. Reed JC: Bcl-2 family proteins. Oncogene 17: 3225-3236, 1998.

6. Joensuu H,Pylkkanen L and Toikkanen S: Bcl-2 protein expression and long-term survival in breast cancer. Am J Pathol 145: 1191-1198, 1994.

7. Sinicrope FA, Ruan SB, Cleary KR, Stephens LC, Lee JJ and Levin B: Bcl-2 and p53 oncoprotein expression during colorectal tumorigenesis. Cancer Res 55: 237-241, 1995.

8. Ligueros M, Jeoung D, Tang B, Hochhauser D, Reidenberg MM and Sonenberg M: Gossypol inhibition of mitosis, cyclin D1 and $\mathrm{Rb}$ protein in human mammary cancer cells and cyclin-D1 transfected human fibrosarcoma cells. Br J Cancer 76: 21-28, 1997.

9. Jiang J, Kulp SK, Sugimoto Y, Liu S and Lin YC: The effects of gossypol on the invasiveness of MAT-LyLu cells and MAT-LyLu cells from the metastasized lungs of MAT-LyLu-bearing Copenhagen rats. Anticancer Res 20: 4591-4597, 2000. 
10. Ready N, Karaseva NA, Orlov SV, Luft AV, Popovych O, Holmlund JT, Wood BA and Leopold L: Double-blind, placebo-controlled, randomized phase 2 study of the proapoptotic agent AT-101 plus docetaxel, in second-line non-small cell lung cancer. J Thorac Oncol 6: 781-785, 2011.

11. Sonpavde G, Matveev V, Burke JM, Caton JR, Fleming MT, Hutson TE, Galsky MD, Berry WR, Karlov P, Holmlund JT, et al: Randomized phase II trial of docetaxel plus prednisone in combination with placebo or AT-101, an oral small molecule Bcl-2 family antagonist, as first-line therapy for metastatic castration-resistant prostate cancer. Ann Oncol 23: 1803-1808, 2012.

12. Baggstrom MQ, Qi Y, Koczywas M, Argiris A, Johnson EA, Millward MJ, Murphy SC, Erlichman C, Rudin CM, Govindan R, et al: A phase II study of AT-101 (Gossypol) in chemotherapy-sensitive recurrent extensive-stage small cell lung cancer. J Thorac Oncol 6: 1757-1760, 2011.

13. Kitada S, Kress CL, Krajewska M, Jia L, Pellecchia M and Reed JC: Bcl-2 antagonist apogossypol (NSC736630) displays single-agent activity in Bcl-2-transgenic mice and has superior efficacy with less toxicity compared with gossypol (NSC19048). Blood 111: 3211-3219, 2008.

14. Hu ZY, Zhu XF, Zhong ZD, Sun J, Wang J, Yang D and Zeng YX: ApoG2, a novel inhibitor of antiapoptotic Bcl-2 family proteins, induces apoptosis and suppresses tumor growth in nasopharyngeal carcinoma xenografts. Int J Cancer 123: 2418-2429, 2008.

15. Niu X, Li S, Wei F, Huang J, Wu G, Xu L, Xu D and Wang S: Apogossypolone induces autophagy and apoptosis in breast cancer MCF-7 cells in vitro and in vivo. Breast Cancer 21: 223-230, 2014

16. Xin J, Zhan Y, Liu M, Hu H, Xia L, Nie Y, Wu K, Liang J and Tian J: ApoG2 induces ER stress-dependent apoptosis in gastric cancer cells in vitro and its real-time evaluation by bioluminescence imaging in vivo. Cancer Lett 336: 260-269, 2013.

17. Cheng P, Ni Z, Dai X, Wang B, Ding W, Rae Smith A, Xu L, Wu D, $\mathrm{He} \mathrm{F}$ and Lian J: The novel BH-3 mimetic apogossypolone induces Beclin-1- and ROS-mediated autophagy in human hepatocellular carcinoma [corrected] cells. Cell Death Dis 4: e489, 2013.

18. Zhang X, Hu X, Mu S, Zhan Y, An Q, Liu Z and Huang X: Apogossypolone inhibits the proliferation of $\mathrm{LNCaP}$ cells in vitro and in vivo. Mol Med Rep 10: 1184-1194, 2014.

19. Sun J, Li ZM, Hu ZY, Zeng ZL, Yang DJ and Jiang WQ: Apogossypolone inhibits cell growth by inducing cell cycle arrest in U937 cells. Oncol Rep 22: 193-198, 2009.
20. Prabhudesai SG, Rekhraj S, Roberts G, Darzi AW and Ziprin P: Apoptosis and chemo-resistance in colorectal cancer. J Surg Oncol 96: 77-88, 2007.

21. Mi JX, Wang GF, Wang HB, Sun XQ, Ni XY,Zhang XW, Tang JM and Yang DJ: Synergistic antitumoral activity and induction of apoptosis by novel pan $\mathrm{Bcl}-2$ proteins inhibitor apogossypolone with adriamycin in human hepatocellular carcinoma. Acta Pharmacol Sin 29: 1467-1477, 2008.

22. Sun Y, Wu J, Aboukameel A, Banerjee S, Arnold AA, Chen J, Nikolovska-Coleska Z, Lin Y, Ling X, Yang D, et al: Apogossypolone, a nonpeptidic small molecule inhibitor targeting Bcl-2 family proteins, effectively inhibits growth of diffuse large cell lymphoma cells in vitro and in vivo. Cancer Biol Ther 7: 1418-1426, 2008

23. Wang X, Wang J, Wong SC, Chow LS, Nicholls JM, Wong YC, Liu Y, Kwong DL, Sham JS and Tsa SW: Cytotoxic effect of gossypol on colon carcinoma cells. Life Sci 67: 2663-2671, 2000.

24. Tuszynski GP and Cossu G: Differential cytotoxic effect of gossypol on human melanoma, colon carcinoma and other tissue culture cell lines. Cancer Res 44: 768-771, 1984.

25. Lombardi L, Frigerio S, Collini P, and Pilotti S: Immunocytochemical and immunoelectron microscopical analysis of BCL2 expression in thyroid oxyphilic tumors. Ultrastruct Pathol 21: 33-39, 1997.

26. Wei J, Stebbins JL, Kitada S, Dash R, Zhai D, Placzek WJ, Wu B, Rega MF, Zhang Z, Barile E, et al: An optically pure apogossypolone derivative as potent pan-active inhibitor of antiapoptotic bcl-2 family proteins. Front Oncol 1: 28, 2011.

27. Weng C, Li Y, Xu D, Shi Y and Tang H: Specific cleavage of Mcl-1 by caspase-3 in tumor necrosis factor-related apoptosis-inducing ligand (TRAIL)-induced apoptosis in Jurkat leukemia T cells. J Biol Chem 280: 10491-10500, 2005.

28. Hernandez JM, Farma JM, Coppola D, Hakam A, Fulp WJ, Chen DT, Siegel EM, Yeatman TJ and Shibata D: Expression of the antiapoptotic protein survivin in colon cancer. Clin Colorectal Cancer 10: 188-193, 2011.

29. Chen J, Chen Y and Chen Z: MiR-125a/b regulates the activation of cancer stem cells in paclitaxel-resistant colon cancer. Cancer Invest 31: 17-23, 2013.

30. Poincloux L, Durando X, Seitz JF, Thivat E, Bardou VJ, Giovannini MH, Parriaux D, Barriere N, Giovannini M, Delpero JR and Monges G: Loss of Bcl-2 expression in colon cancer: A prognostic factor for recurrence in stage II colon cancer. Surg Oncol 18: 357-365, 2009. 